\title{
Reversed Stereoselectivity in Iodohydroxylation of Allenyl Sulfides. An Efficient Synthesis of Z-3-Organosulfur-2-iodo-2-propenols
}

\author{
Shengming Ma,* Xueshi Hao, and Xian Huang
}

\section{Supporting Information}

\author{
Experimental section
}

Typical procedure for the formation of 2: A solution of allenyl phenyl sulfide(1a) (62 $\mathrm{mg}, 0.25 \mathrm{mmol}$ ) in $1 \mathrm{~mL}$ of $\mathrm{CH}_{3} \mathrm{CN}$ was added slowly to a solution of iodine (127 mg, $0.5 \mathrm{mmol})$ in $\mathrm{CH}_{3} \mathrm{CN}(7 \mathrm{~mL})$ and water $(2 \mathrm{~mL})$ under a $\mathrm{N}_{2}$ atmosphere. After being stirred at $15^{\circ} \mathrm{C}$ for about 16 hours, a saturated aqueous solution of $\mathrm{Na}_{2} \mathrm{~S}_{2} \mathrm{O}_{3}$ was added. The mixture was extracted with ether $(25 \mathrm{~mL}$ x 3$)$ and dried over $\mathrm{MgSO}_{4}$. Evaporation and column chromatography on silica gel (petroleum ether/ethyl acetate $=20: 1$ ) afforded 2a $(21 \mathrm{mg}, 30 \%, E / Z=1.08 / 1)$ as a solid.

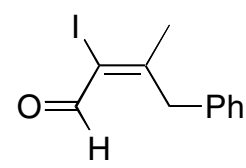

$\boldsymbol{E}$-2-Iodo-3-methyl-4-phenylbut-2-enal (E-2a). solid; m.p. $65-67^{\circ} \mathrm{C}\left(n\right.$-hexane); ${ }^{1} \mathrm{H}$ NMR $\left(300 \mathrm{MHz}, \mathrm{CDCl}_{3}\right) \delta 9.19(\mathrm{~s}, 1 \mathrm{H})$, 7.22-7.27 (m, $\left.3 \mathrm{H}\right)$, 7.07-7.09 (m, $\left.2 \mathrm{H}\right), 4.10(\mathrm{~s}, 2 \mathrm{H})$ 2.15 (s, $3 \mathrm{H}) ;{ }^{13} \mathrm{C}$ NMR $\left(75.4 \mathrm{MHz}, \mathrm{CDCl}_{3}\right) \quad \delta 184.65,165.45,136.34,128.99,128.46$, 127.27, 109.54, 41.10, 32.43; IR (KBr) $\quad\left(\mathrm{cm}^{-1}\right)=3080,3058,3021,2926,2855,1660$, $1585,1575,1492,1448,1396,1369,1224,1185,1089,1073,1030 ; \operatorname{MS}(70 \mathrm{eV}, \mathrm{EI})$ $\mathrm{m} / \mathrm{z}(\%)$ : 286( $\left(\mathrm{M}^{+}, 3.50\right)$, 91(100); HRMS Calcd for $\mathrm{C}_{11} \mathrm{H}_{11} \mathrm{IO}$ : 285.98546. Found: 
285.98692 .

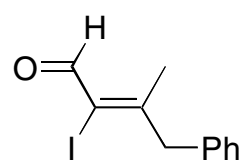

Z-2-Iodo-3-methyl-4-phenylbut-2-enal (Z-2a). solid; m.p. $79-81{ }^{\circ} \mathrm{C}\left(n\right.$-hexane); ${ }^{1} \mathrm{H}$ NMR $\left(300 \mathrm{MHz}, \mathrm{CDCl}_{3}\right) \delta 9.17$ (s, $\left.1 \mathrm{H}\right), 7.24-7.33(\mathrm{~m}, 5 \mathrm{H}), 3.99$ (s, $\left.2 \mathrm{H}\right) 2.33(\mathrm{~s}, 3 \mathrm{H}) ;{ }^{13} \mathrm{C}$ NMR (75.4 MHz, $\left.\mathrm{CDCl}_{3}\right) \delta 185.32,165.22,136.35,128.85,128.73,127.23,108.30$, 52.41, 20.25; IR $(\mathrm{KBr}) \quad\left(\mathrm{cm}^{-1}\right)=3022,1681,1664,1585,1493,1450,1430,1246,1091$, 1071, 1029, 732; $\mathrm{MS}(70 \mathrm{eV}, \mathrm{EI}) \mathrm{m} / \mathrm{z}(\%): 286\left(\mathrm{M}^{+}, 15.65\right), 91(100)$; HRMS Calcd for $\mathrm{C}_{11} \mathrm{H}_{11} \mathrm{IO}: 285.98546$. Found: 285.98558.

Typical procedure for the formation of $Z$-4a: A solution of allenyl phenyl sulfide (64 $\mathrm{mg}, 0.25 \mathrm{mmol})$ in $1 \mathrm{~mL}$ of acetone was added slowly to a solution of iodine $(127 \mathrm{mg}, 0.5$ mmol) in acetone $(7 \mathrm{~mL})$ and water $(2 \mathrm{~mL})$. After being stirred at r.t. for about $13 \mathrm{~h}$, a saturated aqueous solution of $\mathrm{Na}_{2} \mathrm{~S}_{2} \mathrm{O}_{3}$ was added. The mixture was extracted with ether (25 mL x 3) and dried over $\mathrm{MgSO}_{4}$. Evaporation and column chromatography on silica gel (petroleum ether/ethyl acetate $=5: 1)$ afforded Z-4a $(68 \mathrm{mg}, 68 \%)$ as an oil.

\section{(1) Z-3-Iodo-2-methyl-1-phenyl-4-(phenylsulfanyl)but-3-en-2-ol (Z-4a).}

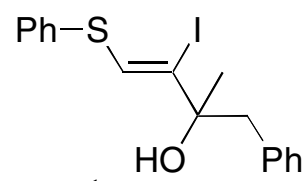

Oil; ${ }^{1} \mathrm{H}$ NMR $\left(300 \mathrm{MHz}, \mathrm{CDCl}_{3}\right)$ ס7.27-7.36 (m, $\left.10 \mathrm{H}\right), 6.80(\mathrm{~s}, 1 \mathrm{H}), 3.21(\mathrm{~d}, J=13.2$ $\mathrm{Hz}, 1 \mathrm{H}), 2.96(\mathrm{~d}, J=13.8 \mathrm{~Hz}, 1 \mathrm{H}), 2.11(\mathrm{~s}, 1 \mathrm{H}) 1.56(\mathrm{~s}, 3 \mathrm{H}) ;{ }^{13} \mathrm{C} \mathrm{NMR}(75.4 \mathrm{MHz}$, $\left.\mathrm{CDCl}_{3}\right) \delta 136.04,134.12,133.70,130.61,130.43,129.11,128.21,127.46,126.93$, 111.26, 77.43, 47.17, 28.00; IR (KBr) $\quad\left(\mathrm{cm}^{-1}\right)=3538,3449,3059,3027,2927,2925$, 
$1581,1568,1478,1452,1440,1372,1341,1239,1113,1090,860, ; \operatorname{MS}(70 \mathrm{eV}, \mathrm{EI})$ m/z(\%): 396( $\mathrm{M}^{+}$, 0.95), 43(100); HRMS Calcd for $\mathrm{C}_{17} \mathrm{H}_{17} \mathrm{IOS}$ : 396.00448. Found: 396.00417.

(2) Z-3-Iodo-4-(phenylsulfanyl)but-3-en-2-ol (Z-4b).

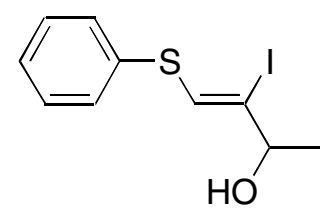

Oil; ${ }^{1} \mathrm{H}$ NMR (300 MHz, $\mathrm{CDCl}_{3}$ ) $87.41-7.44$ (m, $\left.2 \mathrm{H}\right)$, 7.25-7.35 (m, $\left.3 \mathrm{H}\right), 7.07$ (s, $1 \mathrm{H}$ ), $4.03(\mathrm{q}, J=6.0 \mathrm{~Hz}, 1 \mathrm{H}), 2.71(\mathrm{bs}, 1 \mathrm{H}), 1.32(\mathrm{~d}, J=6.0 \mathrm{~Hz}, 3 \mathrm{H}) ;{ }^{13} \mathrm{C} \mathrm{NMR}(75.4 \mathrm{MHz}$, $\left.\mathrm{CDCl}_{3}\right) \delta 133.55,133.35,130.77,129.20,127.67,109.79,73.95,23.77 ; \mathrm{IR}(\mathrm{KBr}) \quad\left(\mathrm{cm}^{-1}\right)$ $=3373,2976,2926,1579,1479,1439,1369,1126,1069,817 ; \mathrm{MS}(70 \mathrm{eV}, \mathrm{EI}) \mathrm{m} / \mathrm{z}(\%):$ 306( $\left.\mathrm{M}^{+}, 49.30\right), 69(100)$; HRMS Calcd for $\mathrm{C}_{10} \mathrm{H}_{11} \mathrm{IOS}: 305.95753$. Found: 305.95662.

(3) Z-2-Iodo-4-methyl-1-(phenylsulfanyl)pent-1-en-3-ol (Z-4c).

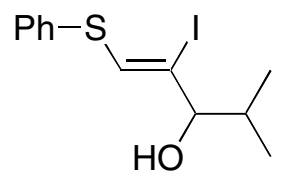

Oil; ${ }^{1} \mathrm{H}$ NMR (300 MHz, $\mathrm{CDCl}_{3}$ ) $87.42-7.45$ (m, $\left.2 \mathrm{H}\right)$, 7.28-7.37 (m, $\left.3 \mathrm{H}\right), 7.04$ (s, $\left.1 \mathrm{H}\right)$, $3.28(\mathrm{~d}, J=8.1 \mathrm{~Hz}, 1 \mathrm{H}), 1.82-1.93(\mathrm{~m}, 2 \mathrm{H}), 1.05(\mathrm{~d}, J=6.1 \mathrm{~Hz}, 3 \mathrm{H}), 0.84(\mathrm{~d}, J=6.9 \mathrm{~Hz}$, $3 \mathrm{H}) ;{ }^{13} \mathrm{C} \mathrm{NMR}\left(75.4 \mathrm{MHz}, \mathrm{CDCl}_{3}\right) \delta 134.63,133.78,130.82,129.28,127.72,108.82$, 83.47, 33.93, 19.16, 17.79; IR (KBr) $\quad\left(\mathrm{cm}^{-1}\right)=3402,2961,2929,2871,1581,1478,1468$, 1440, 1385, 1070, 1025, 864, 809; MS (70 eV, EI) m/z(\%): 334( $\left.\mathrm{M}^{+}, 19.82\right), 291(100)$; HRMS Calcd for $\mathrm{C}_{12} \mathrm{H}_{15} \mathrm{IOS}: 333.98883$. Found: 333.99041.

(4) Z-2-Iodo-1-(phenylsulfanyl)hepta-1-en-3-ol (Z-4d). 


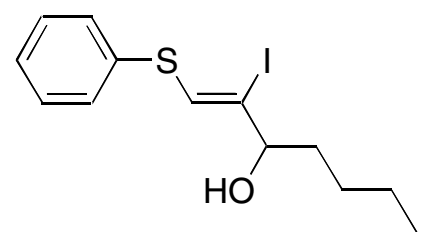

Oil; ${ }^{1} \mathrm{H}$ NMR (300 MHz, $\mathrm{CDCl}_{3}$ ) $87.43-7.45$ (m, $\left.2 \mathrm{H}\right)$, 7.29-7.37 (m, $\left.3 \mathrm{H}\right), 7.06$ (s, $\left.1 \mathrm{H}\right)$, $3.72(\mathrm{t}, J=6.6 \mathrm{~Hz}, 1 \mathrm{H}), 2.08(\mathrm{bs}, 1 \mathrm{H}), 1.62(\mathrm{q}, J=6.9 \mathrm{~Hz}, 2 \mathrm{H}), 1.22-1.39(\mathrm{~m}, 4 \mathrm{H})$, $0.91(\mathrm{t}, J=6.9 \mathrm{~Hz}, 3 \mathrm{H}) ;{ }^{13} \mathrm{C}$ NMR $\left(75.4 \mathrm{MHz}, \mathrm{CDCl}_{3}\right) \delta 134.23,133.72,130.85$, 129.27, 127.74, 109.47, 77.94, 36.86, 29.29, 22.37, 13.97; IR $(\mathrm{KBr}) \quad\left(\mathrm{cm}^{-1}\right)=3375,2955$, 2928, 2857, 1580, 1479, 1465, 1440, 1025, 825; MS(70 eV, EI) m/z(\%): 348( $\left.\mathrm{M}^{+}, 1.28\right)$, 41(100); HRMS Calcd for $\mathrm{C}_{13} \mathrm{H}_{17} \mathrm{IOS}: 348.00448$. Found: 348.00211.

(5) Z-2-Iodo-1-(phenylsulfanyl)dec-1-en-3-ol (Z-4e).

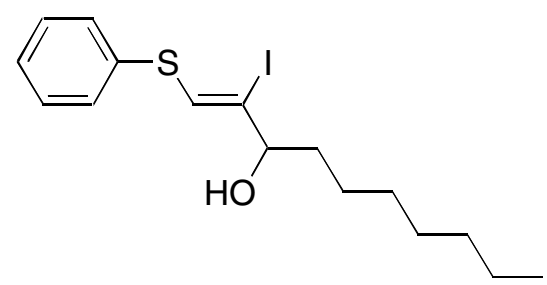

Oil; ${ }^{1} \mathrm{H}$ NMR $\left(300 \mathrm{MHz}, \mathrm{CDCl}_{3}\right)$ 87.42-7.45 (m, $\left.2 \mathrm{H}\right), 7.26-7.37$ (m, $\left.3 \mathrm{H}\right), 7.06(\mathrm{~d}, J=$ $0.6 \mathrm{~Hz}, 1 \mathrm{H}), 3.71(\mathrm{t}, J=6.6 \mathrm{~Hz}, 1 \mathrm{H}), 1.95(\mathrm{bs}, 1 \mathrm{H}), 1.62(\mathrm{q}, J=6.6 \mathrm{~Hz}, 2 \mathrm{H}), 1.22-1.29$ $(\mathrm{m}, 10 \mathrm{H}), 0.88(\mathrm{t}, J=6.6 \mathrm{~Hz}, 3 \mathrm{H}) ; ;{ }^{13} \mathrm{C} \mathrm{NMR}\left(75.4 \mathrm{MHz}, \mathrm{CDCl}_{3}\right) \delta 134.53,134.04$, $131.16,129.57,128.04,109.81,78.26,37.46,32.02,29.53,29.43,25.46,22.89,14.37$; IR $(\mathrm{KBr}) \quad\left(\mathrm{cm}^{-1}\right)=3373,2925,2854,1580,1478,1439,1024,823 ; \mathrm{MS}(70 \mathrm{eV}, \mathrm{EI})$ m/z(\%): 390( $\left.\mathrm{M}^{+}, 24.33\right)$, 43(100); HRMS Calcd for $\mathrm{C}_{16} \mathrm{H}_{23} \mathrm{IOS}$ : 390.05143. Found: 390.05517.

(6) Z-3-Iodo-1-phenyl-4-(phenylsulfanyl)but-3-en-2-ol ( $Z-4 f)$. 


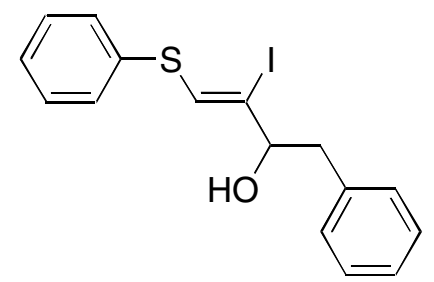

Oil; ${ }^{1} \mathrm{H}$ NMR (300 MHz, CDCl $)$ 87.22-7.36 (m, $\left.10 \mathrm{H}\right), 6.85(\mathrm{~s}, 1 \mathrm{H}), 4.03(\mathrm{t}, J=6.6 \mathrm{~Hz}$, $1 \mathrm{H}), 3.00\left(\mathrm{dd}, J^{\prime}=13.5 \mathrm{~Hz}, \mathrm{~J}^{\prime \prime}=6.3 \mathrm{~Hz}, 1 \mathrm{H}\right), 2.91\left(\mathrm{dd}, J^{\prime}=13.5 \mathrm{~Hz}, \mathrm{~J}^{\prime \prime}=6.9 \mathrm{~Hz}, 1 \mathrm{H}\right)$, 2.15 (bs, $1 \mathrm{H}) ;{ }^{13} \mathrm{C}$ NMR $\left(75.4 \mathrm{MHz}, \mathrm{CDCl}_{3}\right) \delta$ 136.83, 134.72, 133.65, 130.67, 129.55, 129.16, 128.48, 127.60, 126.69, 107.71, 78.97, 43.35; IR $(\mathrm{KBr}) \quad\left(\mathrm{cm}^{-1}\right)=3409,3059$, 3026, 2923, 2855, 1723, 1581, 1478, 1440, 1373, 1247, 1085, 1042, 826; MS(70 eV, EI) $\mathrm{m} / \mathrm{z}(\%)$ : 382( $\left.\mathrm{M}^{+}, 5.59\right), 291(100)$; HRMS Calcd for $\mathrm{C}_{16} \mathrm{H}_{15} \mathrm{IOS}$ : 381.98883. Found: 381.98958.

(7) Z-3-Iodo-2-methyl-4-(phenylsulfanyl)but-3-en-2-ol (Z-4g).

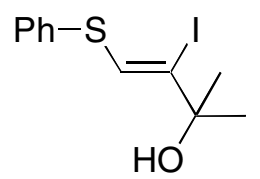

Oil; ${ }^{1} \mathrm{H}$ NMR (300 MHz, $\mathrm{CDCl}_{3}$ ) $87.44-7.47$ (m, $\left.2 \mathrm{H}\right)$, 7.31-7.34 (m, $\left.3 \mathrm{H}\right), 7.03$ (s, $\left.1 \mathrm{H}\right)$, $2.10(\mathrm{bs}, 1 \mathrm{H}), 1.51(\mathrm{~d}, J=1.5 \mathrm{~Hz}, 6 \mathrm{H}) ;{ }^{13} \mathrm{C} \mathrm{NMR}\left(75.4 \mathrm{MHz}, \mathrm{CDCl}_{3}\right) \delta 134.04,132.44$, 130.92, 129.27, 127.71, 113.59, 75.00, 29.68; IR $(\mathrm{KBr}) \quad\left(\mathrm{cm}^{-1}\right)=3403,2979,2928,1582$, 1479, 1440, 1364, 1207, 1167, 1012, 925, 860; MS(70 eV, EI) m/z(\%): 320(M+1 51.98), 305(100); HRMS Calcd for $\mathrm{C}_{11} \mathrm{H}_{13}$ IOS: 319.97318 . Found: 319.97310.

(8) Z-2-Iodo-3-methyl-1-(phenylsulfanyl)pent-1-en-3-ol (Z-4h).

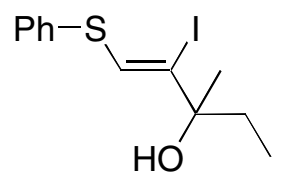

Oil; ${ }^{1} \mathrm{H}$ NMR (300 MHz, $\mathrm{CDCl}_{3}$ ) $87.43-7.46$ (m, $\left.2 \mathrm{H}\right)$, 7.27-7.36 (m, $\left.3 \mathrm{H}\right), 7.05$ (s, $\left.1 \mathrm{H}\right)$, 
$2.10(\mathrm{~s}, 1 \mathrm{H}), 1.67-1.82(\mathrm{~m}, 2 \mathrm{H}), 1.49(\mathrm{~s}, 3 \mathrm{H}), 0.85(\mathrm{t}, J=7.2 \mathrm{~Hz}, 3 \mathrm{H}) ;{ }^{13} \mathrm{C}$ NMR $(75.4$ $\left.\mathrm{MHz}, \mathrm{CDCl}_{3}\right) \delta 134.11,132.92,130.64,129.17,127.50,111.91,77.42,34.53,26.98$, 8.06; IR $(\mathrm{KBr}) \quad\left(\mathrm{cm}^{-1}\right)=3425,2967,2929,2876,1581,1478,1457,1440,1374,1159$, 1121, 1024, 947, 911, 860; MS(70 eV, EI) m/z(\%): 334(M+1 19.13$), 305(100) ;$ HRMS Calcd for $\mathrm{C}_{12} \mathrm{H}_{15} \mathrm{IOS}: 333.98883$. Found: 333.98984 .

(9) Z-2-Iodo-3,5-dimethyl-1-(phenylsulfanyl)hex-1-en-3-ol (Z-4i).

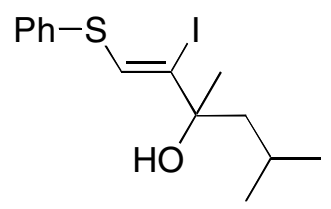

Oil; ${ }^{1} \mathrm{H}$ NMR (300 MHz, $\mathrm{CDCl}_{3}$ ) $87.36-7.39$ (m, $\left.2 \mathrm{H}\right)$, 7.20-7.29 (m, $\left.3 \mathrm{H}\right), 7.01$ (s, $1 \mathrm{H}$ ), $1.76(\mathrm{~s}, 1 \mathrm{H}), 1.60-1.71(\mathrm{~m}, 2 \mathrm{H}), 1.49-1.54(\mathrm{~m}, 1 \mathrm{H}), 1.43(\mathrm{~s}, 3 \mathrm{H}), 0.90$ (d, $J=3.3 \mathrm{~Hz}, 3$ $\mathrm{H}), 0.87(\mathrm{~d}, J=3.3 \mathrm{~Hz}, 3 \mathrm{H}) ;{ }^{13} \mathrm{C} \mathrm{NMR}\left(75.4 \mathrm{MHz}, \mathrm{CDCl}_{3}\right) \delta 134.24,132.52,130.68$, $129.22,127.53,112.47,77.87,49.86,28.87,24.42,24.34,24.17 ; \operatorname{IR}(\mathrm{KBr}) \quad\left(\mathrm{cm}^{-1}\right)=$ $3447,2953,2927,2868,1528,1568,1478,1466,1440,1366,1158,1088,877,855$; MS(70 eV, EI) m/z(\%): 362(M+14.43), 305(100); HRMS Calcd for $\mathrm{C}_{14} \mathrm{H}_{19} \mathrm{IOS}-$ $\mathrm{CH}_{3}\left(\mathrm{C}_{13} \mathrm{H}_{16} \mathrm{IOS}\right):$ 347.99666. Found: 347.00060 .

(10) Z-2-Iodo-3,4,4-trimethyl-1-(phenylsulfanyl)pent-1-en-3-ol (Z-4j).

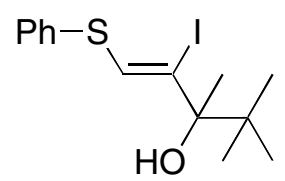

Oil; ${ }^{1} \mathrm{H}$ NMR (300 MHz, $\mathrm{CDCl}_{3}$ ) $87.34-7.37$ (m, $\left.2 \mathrm{H}\right)$, 7.19-7.27 (m, $\left.3 \mathrm{H}\right), 7.01$ (s, $1 \mathrm{H}$ ), $1.79(\mathrm{~s}, 1 \mathrm{H}), 1.47(\mathrm{~s}, 3 \mathrm{H}), 0.98(\mathrm{~s}, 9 \mathrm{H}) ;{ }^{13} \mathrm{C} \mathrm{NMR}\left(75.4 \mathrm{MHz}, \mathrm{CDCl}_{3}\right) \delta 135.92$, $134.37,130.54,129.21,127.44,108.08,79.54,39.41,26.57,26.40 ; \mathrm{IR}(\mathrm{KBr}) \quad\left(\mathrm{cm}^{-1}\right)=$ 3478, 2957, 2872, 1582, 1558, 1479, 1440, 1371, 1073, 901, 861; MS(70 eV, EI) m/z(\%): 
362( $\left.\mathrm{M}^{+}, 1.02\right), 305(100)$; HRMS Calcd for $\mathrm{C}_{14} \mathrm{H}_{19} \mathrm{IOS}: 362.02013$. Found: 362.01818 .

(11) Z-3-Ethyl-2-iodo-1-(phenylsulfanyl)pent-1-en-3-ol (Z-4k).

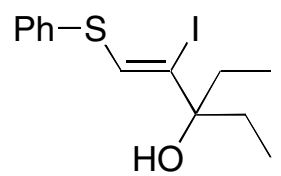

Oil; ${ }^{1} \mathrm{H}$ NMR (300 MHz, $\mathrm{CDCl}_{3}$ ) $87.43-7.46$ (m, $\left.2 \mathrm{H}\right)$, 7.27-7.37 (m, $\left.3 \mathrm{H}\right), 7.08$ (s, $\left.1 \mathrm{H}\right)$, 1.83-1.93 (m, $2 \mathrm{H}), 1.79$ (s, 1H), 1.61-1.71 (m, $2 \mathrm{H}), 0.87$ (t, $J=7.5 \mathrm{~Hz}, 6 \mathrm{H}) ;{ }^{13} \mathrm{C}$ NMR (75.4 MHz, $\left.\mathrm{CDCl}_{3}\right) \delta 134.37,133.47,130.49,129.17,127.38,109.31,79.92,32.59$, 7.42; IR (KBr) $\quad\left(\mathrm{cm}^{-1}\right)=3470,2967,2933,2877,2852,1581,1478,1458,1440,1376$, 1158, 1088, 1024, 961, 865; MS(70 eV, EI) m/z(\%): 348( $\left.\mathrm{M}^{+}, 20.01\right), 319(100)$; HRMS Calcd for $\mathrm{C}_{13} \mathrm{H}_{17} \mathrm{IOS}: 348.00448$. Found: 348.00385 .

(12) Z-2-Iodo-1-(phenylsulfanyl)-3-butylhepta-1-en-3-ol (Z-4l).

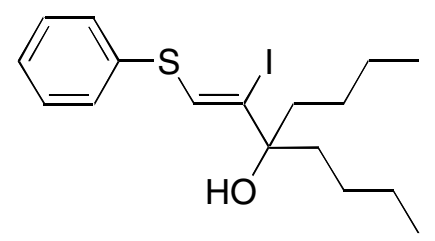

Oil; ${ }^{1} \mathrm{H}$ NMR (300 MHz, $\mathrm{CDCl}_{3}$ ) 87.35-7.38 (m, $\left.2 \mathrm{H}\right)$, 7.19-7.29 (m, $\left.3 \mathrm{H}\right), 6.99$ (s, $\left.1 \mathrm{H}\right)$, 1.73-1.83 (m, 2 H), 1.68 (s, 1H), 1.52-1.58 (m, 2 H), 1.14-1.29 (m, 8 H), 0.84 (t, $J=6.6$ $\mathrm{Hz}, 6 \mathrm{H}) ;{ }^{13} \mathrm{C} \mathrm{NMR}\left(75.4 \mathrm{MHz}, \mathrm{CDCl}_{3}\right) \delta 134.47,132.89,130.47,129.17,127.36$, $110.05,79.59,40.06,25.13,22.82,14.02 ; \mathrm{IR}(\mathrm{KBr}) \quad\left(\mathrm{cm}^{-1}\right)=3475,2955,2870,1723$, 1582, 1570, 1478, 1465, 1440, 1380, 1252, 1089, 865; MS(70 eV, EI) m/z(\%): 404(M+, 4.96), 347(100); HRMS Calcd for $\mathrm{C}_{17} \mathrm{H}_{25} \mathrm{IOS}: 404.06708$. Found: 404.06309.

(13) Z-2-Iodo-1-(phenylsulfanyl)-5-pentylocta-1-en-3-ol (Z-4m). 


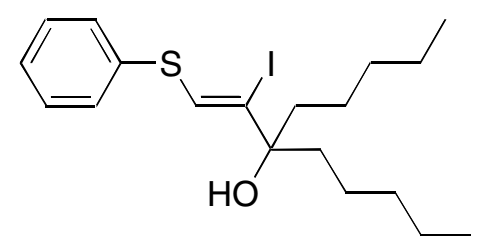

Oil; ${ }^{1} \mathrm{H}$ NMR (300 MHz, $\left.\mathrm{CDCl}_{3}\right)$ ס7.35-7.39 (m, $\left.2 \mathrm{H}\right)$, 7.19-7.30 (m, $\left.3 \mathrm{H}\right), 6.99$ (s, $\left.1 \mathrm{H}\right)$, $1.75-1.80(\mathrm{~m}, 2 \mathrm{H}), 1.66$ (s, $1 \mathrm{H}), 1.49-1.55(\mathrm{~m}, 2 \mathrm{H}), 1.18-1.28(\mathrm{~m}, 12 \mathrm{H}), 0.83(\mathrm{t}, J=6.9$ $\mathrm{Hz}, 6 \mathrm{H}) ;{ }^{13} \mathrm{C} \mathrm{NMR}\left(75.4 \mathrm{MHz}, \mathrm{CDCl}_{3}\right) \delta 134.46,132.81,130.44,129.15,127.33$, $110.19,79.62,40.26,31.92,22.64,22.49,14.04 ; \mathrm{IR}(\mathrm{KBr}) \quad\left(\mathrm{cm}^{-1}\right)=3473,2928,2859$, 1673, 1581, 1478, 1466, 1440, 1378, 1025, 872; MS(70 eV, EI) m/z(\%): 432(M+ 5.36), 361(100); HRMS Calcd for $\mathrm{C}_{19} \mathrm{H}_{29} \mathrm{IOS}: 432.09838$ Found :432.09890.

(14) Z-1-(1'-cyclohexenyl)-1-iodo-2-(phenylsulfanyl)ethene (Z-6n).

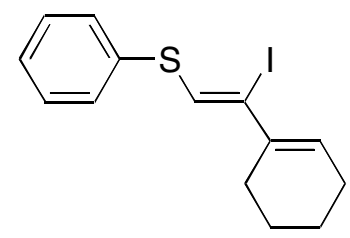

Oil; ${ }^{1} \mathrm{H}$ NMR (300 MHz, $\left.\mathrm{CDCl}_{3}\right)$ 87.47-7.50 (m, $\left.2 \mathrm{H}\right)$, 7.30-7.39 (m, $\left.3 \mathrm{H}\right), 6.91$ (s, $\left.1 \mathrm{H}\right)$, $6.12(\mathrm{t}, J=3.9 \mathrm{~Hz}, 1 \mathrm{H}), 2.31-2.35(\mathrm{~m}, 2 \mathrm{H}), 2.17-2.22(\mathrm{~m}, 2 \mathrm{H}), 1.57-1.70(\mathrm{~m}, 4 \mathrm{H}) ;{ }^{13} \mathrm{C}$ NMR (75.4 MHz, $\left.\mathrm{CDCl}_{3}\right) \delta 135.81,134.59,132.32,131.51,130.52,129.20,127.45$, 105.94, 27.13, 26.15, 22.79, 22.12; MS(70 eV, EI) m/z(\%): 342(M+, 59.10), 218(100); HRMS Calcd forC ${ }_{14} \mathrm{H}_{15}$ IS: 341.99392. Found: 341.99254.

\section{1-(phenylsulfinyl)-2-iodohept-1-en-3-ol (Z-5a).}

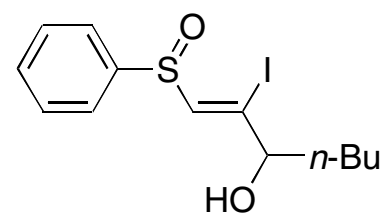

Oil, ${ }^{1} \mathrm{H}$ NMR (300 MHz, $\mathrm{CDCl}_{3}$ ) less polar isomer:ס7.70-7.73 (m, $\left.2 \mathrm{H}\right)$, 7.49-7.53 (m, 3 
H), $7.11(\mathrm{~d}, J=0.9 \mathrm{~Hz}, 1 \mathrm{H}), 4.12-4.15(\mathrm{~m}, 1 \mathrm{H}), 3.51(\mathrm{bs}, 1 \mathrm{H}), 1.73-1.78(\mathrm{~m}, 1 \mathrm{H})$, 1.47-1.54 (m, $1 \mathrm{H}), 1.22-1.30(\mathrm{~m}, 4 \mathrm{H}), 0.81-0.88(\mathrm{~m}, 3 \mathrm{H}) ;{ }^{13} \mathrm{C} \mathrm{NMR}\left(75.4 \mathrm{MHz}, \mathrm{CDCl}_{3}\right)$ $\delta 143.24,140.79,131.33,129.45,124.31,124.28,78.13,35.00,26.89,22.33,13.90$; more polar isomer: $87.22-7.76(\mathrm{~m}, 2 \mathrm{H}), 7.50-7.52(\mathrm{~m}, 3 \mathrm{H}), 7.00(\mathrm{~s}, 1 \mathrm{H}), 3.79(\mathrm{t}, J=$ $12.6 \mathrm{~Hz}, 1 \mathrm{H}), 2.80(\mathrm{bs}, 1 \mathrm{H}), 1.55-1.64(\mathrm{~m}, 2 \mathrm{H}), 1.08-1.27(\mathrm{~m}, 4 \mathrm{H}), 0.81(\mathrm{t}, J=7.2 \mathrm{~Hz}$, $3 \mathrm{H}) ;{ }^{13} \mathrm{C}$ NMR $\left(75.4 \mathrm{MHz}, \mathrm{CD}_{3} \mathrm{COCD}_{3}\right) \delta 145.40,142.60,137.32,131.38,129.71$, 124.28, 77.07, 35.82, 27.15, 22.35, 13.61; IR $(\mathrm{KBr}) \quad\left(\mathrm{cm}^{-1}\right)=3356,2955,2930,2858$, 1582, 1443, 1081, 1030, 997; MS(70 eV, EI) m/z(\%): 364(M+1 , 5.84), 77(100); HRMS Calcd for $\mathrm{C}_{13} \mathrm{H}_{17} \mathrm{IO}_{2} \mathrm{~S}: 363.99940$. Found: 364.00432 . 